\title{
Outcomes of patients with peripheral T-cell lymphoma in first complete remission: data from three tertiary Asian cancer centers
}

\author{
Tiffany Tang1, Lay Poh Khoo', Cindy Lim², Jun Soo Ham, Seok Jin Kim³, Huangming Hong ${ }^{4,5}$, Colin Phipps ${ }^{6}$, \\ Yuh Shan Lee ${ }^{6}$, Miriam Tao', Richard Quek ${ }^{1,7}$, Mohamad Farid', Tongyu Lin' ${ }^{4,5}$, Won Seog Kim ${ }^{3,8}$ and Soon Thye Lim ${ }^{1,7}$
}

Peripheral T-cell lymphomas (PTCL) represent a heterogenous group of aggressive non-Hodgkin's lymphoma, with poorer treatment outcomes compared to that of their B-cell counterparts, using conventional chemotherapy ${ }^{1}$. Despite the lack of randomized data, upfront high-dose chemotherapy (HDC) and autologous stem cell transplantation (ASCT) has been associated with better treatment outcomes ${ }^{2,}{ }^{3}$ and various guidelines now recommend that patients with chemosensitive PTCL should undergo upfront $\mathrm{HDC} / \mathrm{ASCT}^{4}$. Consistently, both retrospective and prospective studies suggest that such a strategy appears to benefit patients with responding disease, especially those in first complete remission (CR1) ${ }^{5}$. However, it is conceivable that PTCL patients who do achieve CR1 may have more favorable survival outcomes, regardless of the treatment they received. Thus, we performed a retrospective analysis of PTCL patients who attained CR1 following first-line induction therapy to determine the factors that would impact their survival outcomes, including the role of upfront HDC/ASCT.

Prospectively maintained T-cell lymphoma databases from the National Cancer Centre Singapore (NCCS)/ Singapore General Hospital (SGH), Samsung Medical Centre, South Korea, and Sun Yat Sen University Cancer Centre, China, were retrospectively reviewed after approval from the institutional review boards of the individual institutions. We included patients with the following histological subtypes: PTCL-not otherwise specified (PTCL-NOS), angioimmunoblastic T-cell

\footnotetext{
Correspondence: Soon Thye Lim (lim.soon.thye@singhealth.com.sg)

'Division of Medical Oncology, National Cancer Centre Singapore, Singapore, Singapore

${ }^{2}$ Division of Clinical Trials and Epidemiological Sciences, National Cancer

Centre Singapore, Singapore, Singapore

Full list of author information is available at the end of the article
}

lymphoma (AITL), anaplastic lymphoma kinase (ALK)negative anaplastic large cell lymphoma (ALCL), and monomorphic epitheliotropic intestinal T-cell lymphoma $(\mathrm{MEITL})^{6,}{ }^{7}$, previously known as type II enteropathic T-cell lymphoma, treated with curative intent. To standardize the effect of treatment on outcome, we excluded patients with ALK-positive ALCL because their first-line treatment would not include considerations for upfront $\mathrm{HDC} / \mathrm{ASCT}^{4}$ and patients with natural-killer/ T-cell lymphoma whose treatment may have included upfront allogeneic stem cell transplantation ${ }^{8}$. Upfront HDC/ASCT was not standard practice in any of the participating institutions and left to the discretion of the primary physician or tumor board decisions. We also excluded patients with composite lymphomas, cutaneous T-cell lymphomas and patients who were not treated with curative intent. We then reviewed the clinical characteristics, treatment and survival outcomes of patients who achieved CR1. Patients who had partial response, stable disease and progression of disease were excluded from the analysis. Treating physicians determined the end of treatment response assessments. The exact modality was as per institutional standards, which was either computed tomography or positron-emission tomography scans.

Progression-free survival (PFS) was defined as the interval from diagnosis to progression, relapse or death. Overall survival (OS) was defined as the interval from diagnosis to death from any cause. Survival estimates were calculated using the Kaplan-Meier method. Survival curves were compared using the log-rank test. Univariate and multivariate analyses were performed using the Cox proportional hazards model to assess the association between several prognostic factors such as age, stage, international prognostic index (IPI) ${ }^{9}$, prognostic index for 
T-cell lymphoma (PIT) ${ }^{10}$ and treatment, including the receipt of upfront HDC/ASCT, with survival outcomes. For multivariate analysis, variable selection was performed using the forward selection method, with selection criteria of $P<0.05$ for inclusion into the final multivariate model. All variables used in the univariate analysis were entered into the forward selection procedure, except for IPI and PIT scores as the individual components of these scores were already included. Two-sided $p$-values less than 0.05 were considered statistically significant. All analyses were performed in Stata (version 14.2, StataCorp, Texas, USA).

There were 114, 96, and 92 patients respectively in the Singapore, South Korea, and China PTCL database. A total of 175 patients were included in our analysis based on the inclusion and exclusion criteria; 57 from Singapore, 62 from South Korea, and 56 from China, resulting in an overall CR1 rate of $57.9 \%$. Patients in the Singapore cohort were diagnosed between October 1998 and December 2015, patients in the Korean cohort were diagnosed between August 2007 and March 2014, and patients in the Chinese cohort were diagnosed between September 2007 and June 2016.

In this study, the median age was 53 years old, $63 \%$ were male and the majority of patients had an ECOG performance status one or less. The most common subtype of PTCL was PTCL-NOS accounting for $42 \%$ of the cohort, followed by AITL 33\%, ALK negative ALCL 22\%, and MEITL 3\%. The majority of patients received anthracycline-based chemotherapy and $18 \%$ underwent upfront HDC/ASCT. Non-anthracycline-based chemotherapy included ICE (ifosfamide, carboplatin, etoposide) (3\%), ESHAP (etoposide, steroids, cytarabine, cisplatin) (1\%), Gemcitabine-based regimens (2\%), and other regimens (2\%) including alemtuzumab-DHAP (steroids, cytarabine, cisplatin) and high-dose methotrexate with procarbazine, and vincristine. In this cohort of patients, the ALK-negative ALCL patients were younger (median age 35 years), they were more likely to have stage I/II disease, normal lactate dehydrogenase (LDH) and less likely to have bone marrow involvement compared to the other subtypes of PTCL in this study. None of the ALK-negative ALCL patients underwent upfront HDC/ASCT (Table 1).

There were several differences between the three cohorts of patients. The Chinese patients were younger (median age 42.5 years), had lower IPI and PIT scores, and they had a significantly higher proportion of patients with ALKnegative ALCL compared to the Singaporean and Korean cohort. With regards to treatment, half of the Chinese patients received $\mathrm{EPOCH}$ as first-line therapy while none of the patients in the Singapore and Korean cohort did, and fewer Chinese patients (7\%) received upfront HDC/ASCT compared to the Singaporean (28\%) and Korean (19\%) cohort. Follow-up times in the Singapore, Korean, and Chinese cohort were also significantly different at 6.1 (95\%

Table 1 Patient characteristics overall and by histological subtype

\begin{tabular}{llllll}
\hline Characteristic & Overall & MEITL & AITL & PTCL- & ALK- \\
& $(n=175)$ & $(n=6)$ & $\begin{array}{l}(n= \\
57)\end{array}$ & $\begin{array}{l}\text { NOS ( } n \\
=74)\end{array}$ & ALCL $(n$ \\
& & & $38)$
\end{tabular}

Age, years

Median

Range

53

Age, years (grouped)

$\leq 60$

$>60$

119 (68)

56 (32)

61.5

5

43-66

28-85

53

35

Sex

Female

65 (37)

$3(50)$

$34(60) \quad 50$ (68)

2 (84)

Male

$110(63)$

2 (33)

$23(40) \quad 24(32) \quad 6(16)$

B symptoms

Present

$76(43)$

$3(50)$

25 (44) 26 (35)

$12(32)$

Absent

92 (53)

2 (33)

$32(56) \quad 48(65) \quad 26(68)$

Unknown

7 (4)

1 (17)

31 (54) 29 (39)

25 (44) 43 (58)

13 (34)

Stage

।

॥

28 (16)

2 (33)

1 (2)

22 (58)

III

$22(13)$

1 (17)

$3(5)$

2 (3)

$3(8)$

IV

57 (33)

0 (0)

1 (2)

16 (22)

7 (18)

61 (35)

2 (33)

26 (46) 19 (26)

$10(26)$

Unknown

7 (4)

$$
27 \text { (47) } 27(36) \quad 5(13)
$$

I / |

III / IV

50 (29)

1 (17)

$0(0)$

2 (3)

$4(11)$

Unknown

$118(67)$

$3(50)$

4 (7)

26 (35)

$17(45)$

53 (93) $46(62) \quad 17(45)$

ECOG performance status

$\begin{array}{llllll}0-1 & 155(89) & 5(83) & 47(82) & 67(91) & 36(95) \\ \geq 2 & 14(8) & 0(0) & 8(14) & 6(8) & 0(0) \\ \text { Unknown } & 6(3) & 1(17) & 2(4) & 1(1) & 2(5)\end{array}$

BM involvement

Yes

$31(18) \quad 0(0)$

$16(28) \quad 14(19) \quad 1(3)$

No

$126(72) \quad 5(83)$

41 (72) 51 (69)

$29(76)$

Unknown

$18(10)$

1 (17)

$0(0)$

9 (12)

8 (21)

Extranodal sites

0-1

$\geq 2$

Unknown

$129(74)$

$4(67)$

43 (75) 56 (76)

26 (68)

$43(25)$

1 (17)

14 (25) $17(23)$

11 (29)

Serum LDH

Elevated

81 (46)

3 (50)

$37(65) \quad 30(41)$

11 (29)

Normal

84 (48)

2 (33)

19 (33) $38(51) \quad 25$ (66) 
Table 1 continued

\begin{tabular}{|c|c|c|c|c|c|}
\hline Characteristic & $\begin{array}{l}\text { Overall } \\
(n=175)\end{array}$ & $\begin{array}{l}\text { MEITL } \\
(n=6)\end{array}$ & $\begin{array}{l}\text { AITL } \\
(n= \\
57)\end{array}$ & $\begin{array}{l}\text { PTCL- } \\
\text { NOS ( } n \\
=74)\end{array}$ & $\begin{array}{l}\text { ALK- } \\
\text { ALCL ( } n \\
=38)\end{array}$ \\
\hline Not evaluated & $10(6)$ & $1(17)$ & $1(2)$ & $6(8)$ & $2(5)$ \\
\hline \multicolumn{6}{|l|}{ IPI score } \\
\hline Low $(0-1)$ & $66(38)$ & $2(33)$ & $12(21)$ & $32(43)$ & $20(53)$ \\
\hline Low-intermediate (2) & $44(25)$ & $1(17)$ & $16(28)$ & $19(26)$ & $8(21)$ \\
\hline High-intermediate (3) & $35(20)$ & $1(17)$ & $18(32)$ & $13(18)$ & $3(8)$ \\
\hline High (4-5) & $12(7)$ & $0(0)$ & $8(14)$ & $4(5)$ & $0(0)$ \\
\hline Not evaluated & $18(10)$ & $2(33)$ & $3(5)$ & $6(8)$ & $7(18)$ \\
\hline \multicolumn{6}{|l|}{ PIT score } \\
\hline Low (0) & $49(28)$ & $2(33)$ & $10(18)$ & $21(28)$ & $16(42)$ \\
\hline Low-intermediate (1) & $55(31)$ & $1(17)$ & $20(35)$ & $25(34)$ & $9(24)$ \\
\hline High-intermediate (2) & $29(17)$ & $1(17)$ & $14(25)$ & $12(16)$ & $2(5)$ \\
\hline High (3-4) & $14(8)$ & $0(0)$ & $10(18)$ & $4(5)$ & $0(0)$ \\
\hline Not evaluated & $28(16)$ & $2(33)$ & $3(5)$ & $12(16)$ & $11(29)$ \\
\hline \multicolumn{6}{|c|}{ Primary treatment regimen } \\
\hline CHOP-like & $133(76)$ & $3(50)$ & $\begin{array}{l}57 \\
(100)\end{array}$ & $53(72)$ & $20(53)$ \\
\hline EPOCH-like & $28(16)$ & $1(17)$ & $0(0)$ & $10(14)$ & $17(45)$ \\
\hline ICE & $6(3)$ & $1(17)$ & $0(0)$ & $4(5)$ & $1(3)$ \\
\hline ESHAP & $1(1)$ & $0(0)$ & $0(0)$ & $1(1)$ & $0(0)$ \\
\hline Gemcitabine-based & $4(2)$ & $1(17)$ & $0(0)$ & $3(4)$ & $0(0)$ \\
\hline Others & $3(2)^{a}$ & $0(0)$ & $0(0)$ & $3(4)^{a}$ & $0(0)$ \\
\hline \multicolumn{6}{|c|}{ Primary treatment regimen (grouped) } \\
\hline Anthracycline based & $161(92)$ & $4(67)$ & $\begin{array}{l}57 \\
(100)\end{array}$ & $63(85)$ & $37(97)$ \\
\hline $\begin{array}{l}\text { Non-anthracycline } \\
\text { based }\end{array}$ & $14(8)$ & $2(33)$ & $0(0)$ & $11(15)$ & $1(3)$ \\
\hline Others & $0(0)$ & $0(0)$ & $0(0)$ & $0(0)$ & $0(0)$ \\
\hline \multicolumn{6}{|l|}{ Auto-SCT } \\
\hline Yes & $32(18)$ & $1(17)$ & $16(28)$ & $15(20)$ & $0(0)$ \\
\hline No & $143(82)$ & $5(83)$ & $41(72)$ & $59(80)$ & $38(100)$ \\
\hline
\end{tabular}

aalemtuzumab-DHAP; High-dose methotrexate/procarbazine/vincristine

confidence interval (CI), 4.3-7.6), 3.0 (95\% CI, 2.2-4.1), and 1.6 (95\% CI 1.1-2.0) years respectively.

For the entire cohort, the median OS was not reached and the median PFS was 5.5 years ( $95 \% \mathrm{CI}, 4.0-8.2$ years). The 3-year OS and PFS rates were 89\% (95\% CI, 82-93\%) and $61 \%$ (95\% CI, 52-68\%), respectively and the median follow-up time was 2.7 years ( $95 \% \mathrm{CI}, 2.2-3.2$ years). The 3-year OS for ALK- negative ALCL was 100\% compared to $89 \%$ (95\% CI $76-95 \%)$ in PTCL-NOS, $84 \%$ (95\% CI 70-02\%) in AITL, and 80\% (20-97\%) in MEITL.
On univariate analysis, older age, higher stage, IPI, and PIT scores were significantly associated with a poorer OS. Histological subtypes also significantly correlated with OS on univariate analysis. Age, stage, IPI, and PIT scores, gender and treatment site correlated with PFS on univariate analysis. On multivariate analysis, older age and higher stage were independently prognostic for a poorer OS and older age, male gender and higher stage were independently prognostic for a poorer PFS. Interestingly, there was no evidence that primary treatment, i.e., anthracycline versus non-anthracycline-based treatment or upfront HDC/ASCT correlated with PFS or OS on univariate and multivariate analyses. Even when we considered only patients below 60 years of age, upfront HDC/ ASCT did not appear to be associated with a better OS or PFS (Table 2).

We further analyzed the data excluding patients with ALK-negative ALCL since they were a low-risk population that did not undergo upfront HDC/ASCT in this study and found that an older age, male gender, advanced stage, higher PIT scores, and upfront HDC/ASCT were associated with a poorer OS on univariate analysis. However, on multivariate analysis, only age and gender were significantly associated with a poorer OS. Excluding the ALK-negative cohort, advanced age, male gender, advanced stage, elevated LDH, higher IPI, and PIT scores were associated with a poorer PFS on univariate analysis but only male gender and advanced stage were significantly associated with a poorer PFS on multivariate analysis. We also performed an analysis excluding the Chinese cohort of patients as they appeared to have a cohort of patients with more favorable parameters including a younger age, lower IPI, and PIT scores and fewer Chinese patients received upfront HDC/ASCT. In this analysis, a higher age, stage, PIT scores, and the male gender were associated with a poorer OS on univariate analysis and only a higher age and stage were significantly associated with a poorer OS on multivariate analysis. Excluding the Chinese cohort, a higher age and stage, and the male gender were associated with a poorer PFS on both univariate and multivariate analyses.

The retrospective nature of this study means patient and treatment factors, such as first-line chemotherapy regimens, are not standardized. Data on dose intensity and density are also limited. Although most of the histological diagnoses were made by hematopathologists at tertiary academic centers, there was no central review and diagnoses were made according to the World Health Organization (WHO) guidelines, which have undergone changes over the years. Small numbers of patients also limits the subgroup analysis and the manner in which end of treatment assessments were performed was variable according to institutional standards. Nonetheless, given the rarity of this disease, this study provides valuable 


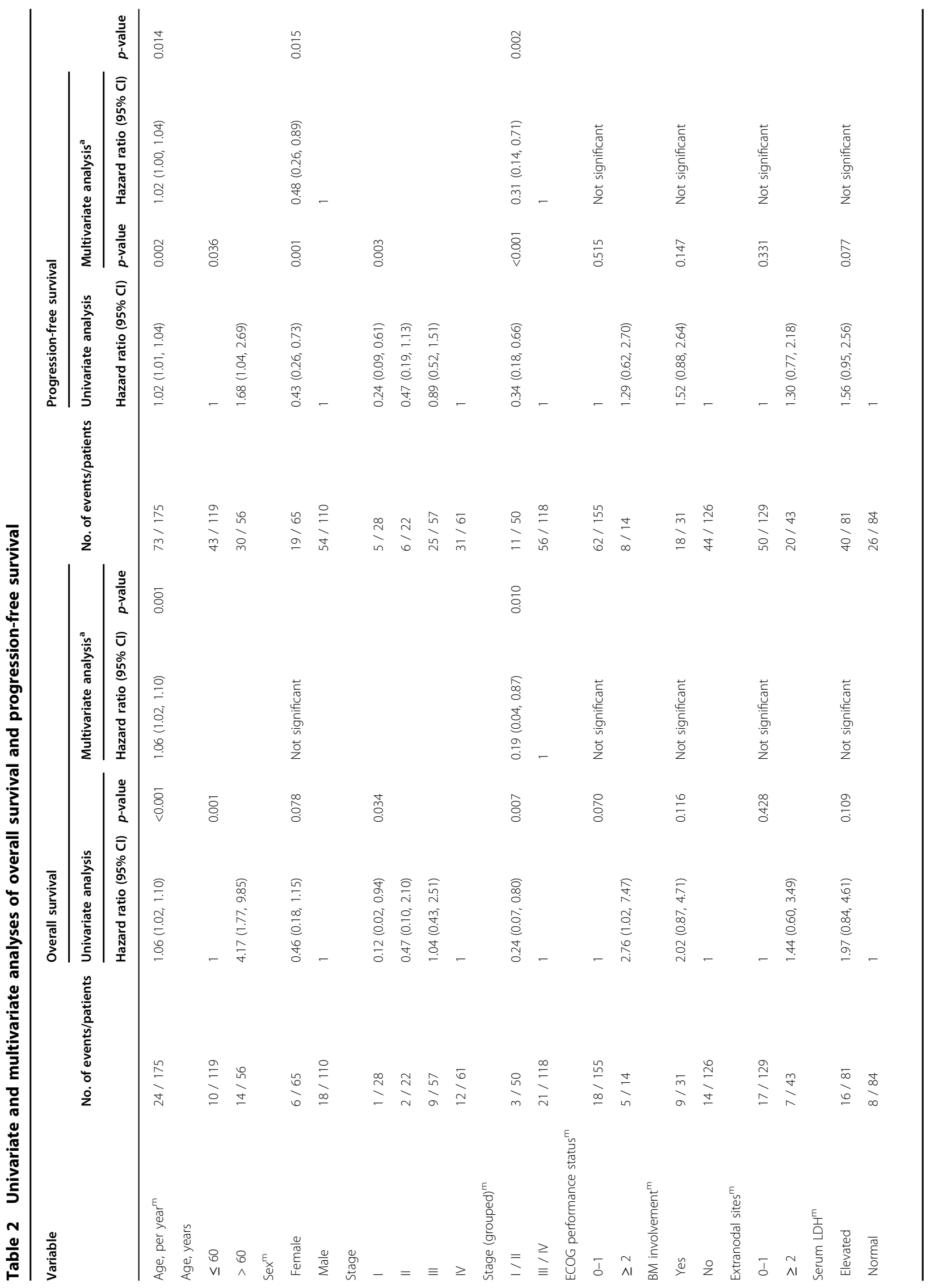




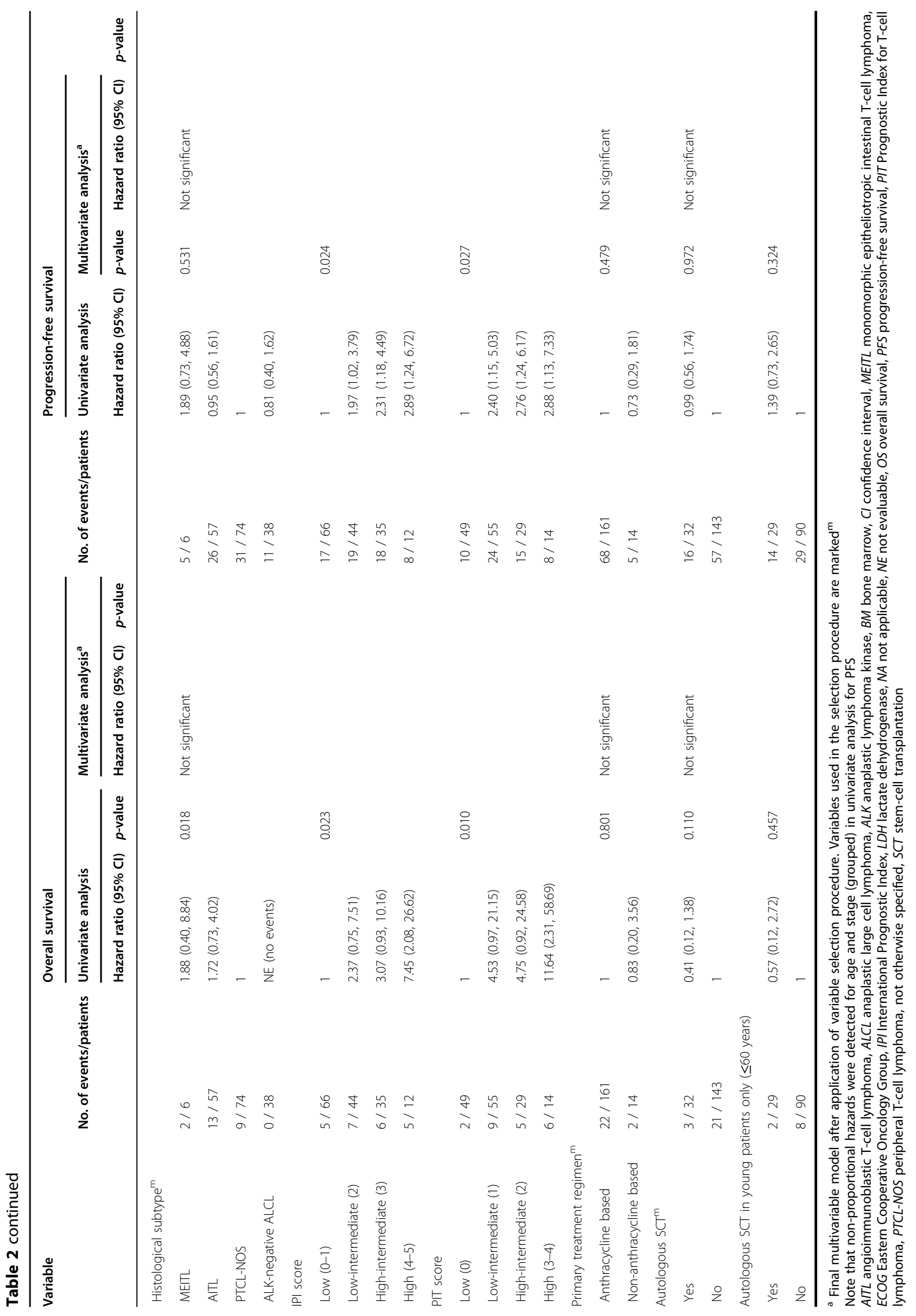


information from large and longitudinal data sets from three major academic centers in Asia and it reflects realworld experience.

In conclusion, our study shows that patients with PTCL who do achieve CR1 may have a better prognosis than their contemporaries who do not achieve CR1. Similar to other reports $^{11-13}$, the use of HDC/ASCT in our study did not appear to improve PFS or OS. Prospective trials are needed to validate this observation.

\section{Author details}

'Division of Medical Oncology, National Cancer Centre Singapore, Singapore, Singapore. ${ }^{2}$ Division of Clinical Trials and Epidemiological Sciences, National Cancer Centre Singapore, Singapore, Singapore. ${ }^{3}$ Division of HematologyOncology, Department of Medicine, Samsung Medical Center, Sunkyunkwan University School of Medicine, Seoul, Korea. ${ }^{4}$ Department of Medical Oncology, Sun Yat-Sen University Cancer Center, Guangzhou, People's Republic of China. ${ }^{5}$ State Key Laboratory of Oncology in Southern China, Collaborative Innovation Center of Cancer Medicine, Sun Yat-Sen University Cancer Centre Guangzhou, Guangzhou, People's Republic of China. 'Department of Hematology, Singapore General Hospital, Singapore, Singapore. ${ }^{7}$ Oncology Academic Clinical Program, Duke-NUS Graduate Medical School, Singapore, Singapore. ${ }^{8}$ Department of Health Sciences and Technology, SAIHST, Sunkyunkwan University, Seoul, Korea

\section{Competing interests}

The authors declare that they have no competing interests.

\section{Publisher's note}

Springer Nature remains neutral with regard to jurisdictional claims in published maps and institutional affiliations.

Received: 11 September 2017 Revised: 14 October 2017 Accepted: 20 October 2017

Published online: 15 December 2017

\section{References}

1. Vose, J., Armitage, J. \& Weisenburger, D. International TCLP. International peripheral T-cell and natural killer/T-cell lymphoma study: pathology findings and clinical outcomes. J. Clin. Oncol. 26, 4124-4130 (2008).

2. d'Amore, F. et al. Up-front autologous stem-cell transplantation in peripheral T-cell lymphoma: NLG-T-01. J. Clin. Oncol. 30, 3093-3099 (2012).

3. Ellin, F., Landstrom, J., Jerkeman, M. \& Relander, T. Real-world data on prognostic factors and treatment in peripheral T-cell lymphomas: a study from the Swedish Lymphoma Registry. Blood 124, 1570-1577 (2014).

4. d'Amore, F. et al. Peripheral T-cell lymphomas: ESMO Clinical Practice Guidelines for diagnosis, treatment and follow-up. Ann. Oncol. 26(Suppl 5), v108-v115 (2015)

5. Casulo, C. \& Horwitz, S. Should eligible patients with T-cell lymphoma receive high-dose therapy and autologous stem cell transplant in the upfront setting? Curr. Oncol. Rep. 12, 374-382 (2010).

6. Swerdlow, S. H. et al. The 2016 revision of the World Health Organization classification of lymphoid neoplasms. Blood 127, 2375-2390 (2016).

7. Tan, S. Y. et al. Type II EATL (epitheliotropic intestinal T-cell lymphoma): a neoplasm of intra-epithelial T-cells with predominant CD8alphaalpha phenotype. Leukemia 27, 1688-1696 (2013).

8. Tse, E. \& Kwong, Y. L. How I treat NKT-cell lymphomas. Blood 121, 4997-5005 (2013).

9. Shipp, M. A predictive model for aggressive non-Hodgkin's lymphoma. The International Non-Hodgkin's Lymphoma Prognostic Factors Project. N. Eng. J. Med. 329, 987-994 (1993).

10. Gallamini, A. et al. Peripheral T-cell lymphoma unspecified (PTCL-U): a new prognostic model from a retrospective multicentric clinical study. Blood 103 2474-2479 (2004).

11. Yam, C. et al. Autologous stem cell transplantation in first complete remission may not extend progression-free survival in patients with peripheral T cell lymphomas. Am. J. Hematol. 91, 672-676 (2016).

12. Cederleuf, H. et al. Outcome of peripheral T-cell lymphoma in first complete remission: a Danish-Swedish population-based study. Leuk. Lymphoma. $\mathbf{5 8}$ 2815-2823 (2017)

13. Abramson, J. S. et al. Peripheral T-cell lymphomas in a large US multicenter cohort: prognostication in the modern era including impact of frontline therapy. Ann. Oncol. 25, 2211-2217 (2014). 